\title{
Healthy eating advice during pregnancy lacks depth: the views of midwives and service users
}

Pregnancy offers opportunity to improve individual eating patterns. During pregnancy many women become more aware of health; seeking clear, credible, evidence-based information ${ }^{(1)}$. NICE recommends that all women presenting for antenatal care should receive healthy eating advice from midwives ${ }^{(2)}$. However, given the complexity of issues surrounding healthy eating, midwives may have difficulties incorporating such advice into their appointments. The aim of these studies was to explore the experiences of midwives and service users regarding the translation of healthy eating advice to pregnant women.

Midwives and pregnant/postnatal women were recruited from various community locations in Liverpool and Ulster. Semi-structured interviews were conducted with 17 midwives $(n=9$ Liverpool, $n=8$ Ulster) and 31 service users, via four focus groups; ( 2 in Liverpool $n=10 \& 5$ and 2 in Ulster $n=7 \& 9)$. Group discussions and reflective notes were transcribed and subjected to Thematic Analysis ${ }^{(3)}$.

A master theme identified from both the midwives and the service users' data, was that healthy eating advice - whilst important currently lacked consistency and depth.

Midwives acknowledged that they should give healthy eating advice, but none had received any education to support this, hence this created uncertainty and thus variation in any advice offered:

"We don't have any training ... or updates or anything about diet in pregnancy."

Service users acknowledged such limitations, reflecting directly on the advice they did / did not receive:

"Some of them just weren't interested in nutrition at all. It wasn't helpful at all. Maybe it was lack of knowledge as well; they maybe didn't feel comfortable \& were just skimming over it".

When offered, most midwives described healthy eating in rather general terms, recommending to 'cut out junk food', 'make healthy choices' and/or 'cut out fat and oil'.

Service users reported this negatively and wanted to know what they should do, rather than focusing on food to avoid:

"They told me not to be eating a lot of eggs, \& what to limit, like food safety, liver \& soft cheeses - that sort of thing".

Service users recalled midwives asking about supplement use, but they did not expand on dietary sources of micronutrients:

"They asked did I take vitamins but didn't really give more knowledge about the foods to get them from".

Women were motivated to make dietary changes during pregnancy but reported insufficient support to do so. Both midwives and service users identified that a lack of in-depth dietary knowledge was a barrier to providing this support, and midwives felt that inadequate training was responsible. Involving nutrition and psychology experts in the education of midwives would enable them to offer more in-depth, positive healthy eating messages; more likely to meet the needs and expectations of service users.

1. Olander E, Atkinson L, Edmunds JK, French DP (2012) Prim Health Care Res Dev, 237-243.

2. NICE (2016) Quality Standard, 22.

3. Braun, V., Clarke, V., Terry, G. (2014) Qualitative research in clinical \& health psychology: MacMillan. 